\title{
Case report special
}

\section{Transrectal ultrasonographic diagnosis of jejunoileal intussusception in a cow}

\author{
Tolga Karapinar and Mustafa Kom \\ Faculty of Veterinary Medicine, Firat University, 23119, Elazig, Turkey \\ Corresponding author: Tolga Karapinar. Tel: +90 04242370000 (ext: 3872) \\ Email: tolgakarapinar@yahoo.com
}

Irish Veterinary Journal Volume 60 Number 7, 422-424, 2007

Intussusception is defined as the invagination of a portion of bowel into the lumen of the adjacent segment of bowel (Smith, 1985). Intussusception, one of the causes of intestinal obstruction, is not a common gastrointestinal condition in cattle (Pearson and Pinsent, 1977; Smith, 1985; Smith, 1990) and is usually seen in calves less than two months old (Constable et al., 1997). The cause of an intussusception is often unknown but any condition that alters intestinal motility has been implicated (Aytug, 1991; Constable et al., 1997). It is suggested that intussusception can represent a diagnostic challenge in cattle medicine. History, clinical and clinicopathologic examinations are important in the diagnosis of intussusception (Constable, et. al, 1997; Smith, 1984). Although ultrasonography has been commonly used as a diagnostic tool to detect internal diseases of large animals, there is limited ultrasonographic data demonstrating intussusceptions in cattle (Braun et al., 1995; Edens et al., 1996; Braun, 2003). This case report describes a small intestinal intussusception in a cow and the transrectal ultrasonographic view of the lesion.

In November 2005, a four-year-old, non-pregnant Simmental cow, with a history of anorexia, colic and a lack of defaecation was submitted to the clinic after two days without treatment. Values for rectal temperature, pulse and respiratory rate were $38.2{ }^{\circ} \mathrm{C}, 100$ beats/minute and 20 breaths/minute, respectively. Ruminal contractions were markedly reduced (two contractions every five minutes). The cow showed mild abdominal pain. Auscultation with percussion over the right flank was negative and abdominal distention was not evident. Rectal examination revealed the presence of a firm mass on the left of the median line and cranial to the pelvis. There was no faecal material in rectum. No distended loops of small intestine were palpable on rectal examination. The firm mass was examined using an $8-\mathrm{MHz}$ rectal transducer (100 Falco, Pie Medical). The transducer was placed in a rectal palpation sleeve containing transducer coupling gel and inserted per rectum. The longitudinal ultrasound view of the firm mass revealed several echogenic parallel densities with a hypoechogenic central core representing the intestinal lumen of the intussusception (Figure 1). Ultrasonographic examination of the right flank failed to reveal dilated loops of small

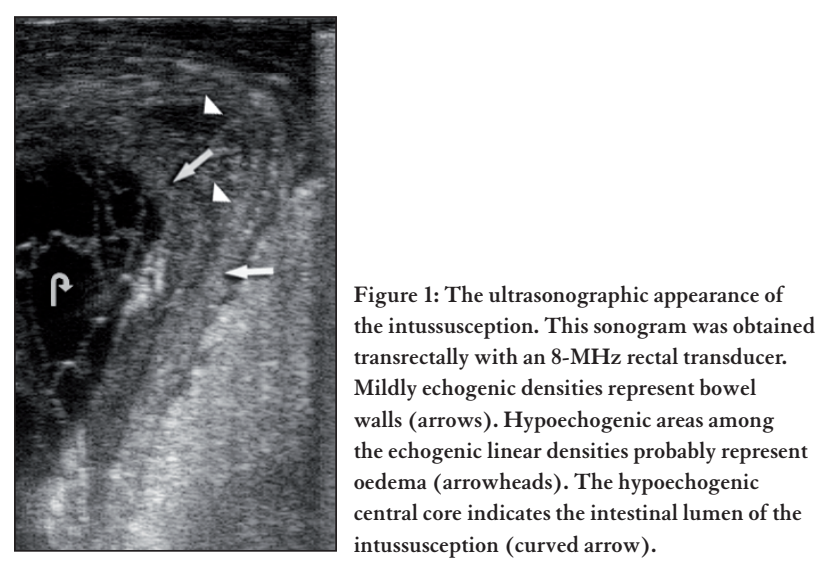

intestine.

The cow was slightly dehydrated. Haematocrit value, total serum protein and fibrinogen concentrations were $36 \%$ (reference range $24-46 \%$ ), $7 \mathrm{~g} / \mathrm{dl}$ (reference range $6.7-7.5 \mathrm{~g} / \mathrm{dl}$ ), and $600 \mathrm{mg} / \mathrm{dl}$ (reference range 100-600 $\mathrm{mg} / \mathrm{dl})$, respectively. Clinical pathology revealed that there was a mild hyperglycemia $(5.1645 \mathrm{mmol} / \mathrm{L}$; reference range $2.4975-4.1625 \mathrm{mmol} / \mathrm{L})$, hypocalcaemia (1.675 $\mathrm{mmol} / \mathrm{L}$; reference range $2.425-3.1 \mathrm{mmol} / \mathrm{L}$ ) and a slight hypochloraemia (93 mmol/L; reference range $97-111$ $\mathrm{mmol} / \mathrm{L})$. Serum urea, creatinine, potassium and sodium concentrations were all within the normal range.

Based on the history and the results of physical, ultrasonographic and biochemical examination, a tentative diagnosis of intussusception was made and the cow was submitted for surgery. Surgery was performed by a right flank laparotomy with the cow in a standing position, under local infiltration analgesia using $2 \%$ lidocaine hydrochloride (Vilcain; Vilsan, Ankara, Turkey). The intussusception was found. However, it could not be reduced by manual traction. An end-to-end anastomosis was performed following the jejunoileal resection. Postoperative therapy included $0.9 \%$ sodium chloride (10 L iv, daily) (Eczacibasi-Baxter, Istanbul, Turkey) for two days and penicillin (Strepto-Veticilline; Eczacibasi-Baxter, Istanbul, Turkey) for five days. The cow became appetent one day post surgery and made steady improvement over the following ten days. The resected bowel segment (Figure 
2) was placed in formalin for subsequent histopathological examination. Histopathological examination confirmed that the intussusceptum was distal jejunum and that the intussucipiens was ileum.

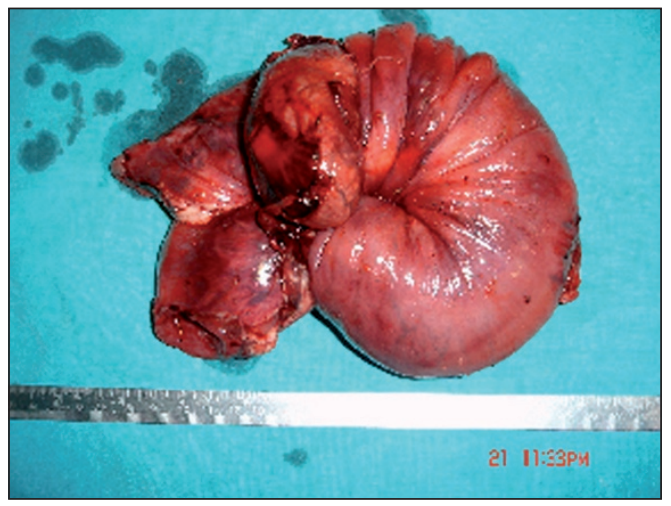

Figure 2: The view of the resected bowel segment.

Different types of intussusception have been recognised in cattle such as enteric, ileoileal, ileocecocolic, cecocolic and colocolic. Among these types, the enteric type of intussusception which occurs with invagination of one segment of the small intestine into another (usually distal jejunum or the ileum), is the most common form in cattle (Pearson, 1971; Constable et al., 1997). Enteric intussusception was diagnosed in this case. Although the majority of intussusceptions are idiopathic, intussusception may result from enteritis, intestinal parasitism, abrupt dietary changes, drug-induced changes in intestinal motility, mural or luminal intestinal lesions and foreign bodies (Ein and Stephens, 1971; Pearson, 1971; Robertson, 1979; Lewis and Ellison, 1987; Ford et al., 1990; Constable et al., 1997). The prevalence of enteric intussusception in cattle has been attributed to the length and mobility of the jejunal mesenteric attachments, especially the distal third (Smith, 1985). Based on history, clinical examination findings on laparotomy and pathological findings, the cause of intussusception couldn't be determined in this case.

Classic signs of intussusception are, initially, either chronic low grade pain or acute signs of abdominal pain, followed by progressive inappetence, abdominal distention, a reduction in faecal volume and lethargy (Smith, 1980). The cow showed slight signs of colic, anorexia, lack of defaecation and lethargy but no abdominal distention. In the diagnosis of small intestinal obstruction, rectal palpation is considered valuable. Although intussusception is palpable in only a minority of affected adult cattle (23\%), distended loops of small intestine are palpable per rectum in $50 \%$ of cases with intussusception (Constable, et. al., 1997). In this case, while the intussusception was diagnosed on the rectal examination, neither rectal palpation nor ultrasonographic examination of the right flank revealed distended loops of small intestine.

The transabdominal ultrasonographic pattern of an intussusception in cross-section has been described as 'bowel within bowel', a 'bull's eye' lesion, 'target pattern' or as a multiple layered, 'onion ring-type' mass with varying echogenities (Bernard et al., 1989; Braun, 2003). In longitudinal section, the typical lumen-within-a-lumen can be clearly identified and has been described as a 'sandwich' configuration (Braun, 2003). Abdominal ultrasonography was attempted using a $3.5 \mathrm{MHz}$ curved array with a maximal penetration depth of $17 \mathrm{~cm}$, but the lesion could not be imaged probably because of the depth of the lesion.

Transrectal ultrasonography has also been employed to diagnose intussusceptions in equine medicine. The transrectal ultrasonographic pattern associated with the intussusception varies in respect to transducer placement. It has been suggested that while the targetlike configuration is obtained on a transverse section of intussusception, longitudinal ultrasound views of the intussusception reveals several echogenic parallel densities and a hypoechogenic core (Edens et al., 1996). In the present case, there were several echogenic parallel densities with a hypoechogenic central core representing the intestinal lumen of the intussusception. Moreover, hypoechogenic densities were evident in parallel with hyperechogenic areas. The transrectal ultasonographic findings of the case are similar to those described by Edens et al. (1996). The hyperechogenic densities indicate the bowel walls. Hypoechogenic parallel linear densities interspersed among hyperechogenic areas probably correspond to oedema. Lack of faecal output, signs of abdominal pain, the palpation of a firm mass on rectal examination and especially the transrectal ultrasonographic pattern of the lesion led to the suspect diagnosis of intussusception requiring immediate exploratory laparotomy. Small intestinal intussusceptions are surgically repaired by means of resection and end-toend anastomosis in both cattle and horses (Bernard et al., 1989; Constable et al., 1997; Dabak et al., 2001; FontaineRodgerson and Rodgerson, 2001), because end-to-end anastomosis causes less chance of stricture formation and leakage (Constable et al., 1997). This technique was preferred in this cow and no complications were observed. In conclusion, transrectal ultrasonography can be a valuable diagnostic tool in cattle that have a palpable mass on rectal examination.

\section{Acknowledgements}

We express our deep appreciation to Associate Professor Murat Dabak and Professor M. Resat Ozercan.

\section{References}

Aytug, C.N. (1991). Sigir Hastaliklari. Istanbul: Tumvet. Bernard, W.V., Reef V.B., ReimeR J.M., Humber, K.A. and Orsini, J.A. (1989). Ultrasonographic diagnosis of small-intestinal intussusception in three foals. Journal of the American Veterinary Medical Association 194: 395 397.

Braun, U. (2003). Ultrasonography in gastrointestinal disease in cattle. The Veterinary Journal 166: 112-124.

Braun, U., Pusterla, N. and Wild, K. (1995). Ultrasonographic findings in 11 cows with a hepatic abscess. Veterinary Record. 137: 284-290.

Constable, P.D., StJean, G., Hull, B.L., Rings, D.M., Morin, D.E. and 
Nelson, D.R. (1997). Intussusception in cattle: 336 cases (1964-1993). Journal of the American Veterinary Medical Association 210: 531-536. Dabak, M., Unsaldi, E. and Gul, Y. (2001). Diagnosis and treatment of intussusception in a cow. Turkish Journal of Veterinary Animal Science 25: 387-389.

Edens, L.M., White, N.A., Dabareiner, R.M. and Sullins, K.E. (1996).

Transrectal ultrasonographic diagnosis of an ileocaecal intussusception in a horse. Equine Veterinary Journal 28: 81-83.

Ein, S.H. and Stephens C.A. (1971). Intussusception: 354 cases in 10 years. Journal of Pediatric Surgery 6: 16-27.

Fontaine-Rodgerson, G. and Rodgerson, D.H. (2001). Diagnosis of small intestinal intussuception by transabdominal ultrasonography in 2 adult horses. Canadian Veterinary Journal 42: 378-380.

Ford, T.S., Freeman, D.E., Ross, M.W., Richardson, D.W., Martin, B.B. and Madison, J.B. (1990). Ileocecal intussusception in horses: 26 cases (1981-1988). Journal of the American Veterinary Medical Association 196: 121-126.
Lewis, D.D. and Ellison, G.W. (1987). Intussusceptions in dogs ang cats. Compendium on Continuing Education for the Practicing Veterinarian 9: 523-533

Pearson, H. and Pinsent, H,J. (1977). Intestinal obstruction in cattle. Veterinary Record. 101: 162-166.

Smith, D.F. (1980). Intussusception in adult cattle. Compendium on Continuing Education for the Practicing Veterinarian - Large Animal Supplements 11: S49-S53.

Smith, D.F. (1984). Bovine intestinal surgery: Part 3. Modern Veterinary Practice 65: 909-912.

Smith, D.F. (1985). Bovine intestinal surgery: Part 5. Modern Veterinary Practice 66: 405-409.

Smith D.F. (1990). Surgery of the bovine small intestine. Veterinary Clinics of North America Food Animal Practice 6: 449-60.

Pearson, H. (1971). Intussusception in cattle. Veterinary Record 89: 426-437. Robertson, J.T. (1979). Differential diagnosis and surgical management of intestinal obstruction in cattle. Veterinary Clinics of North America Large Animal Practice 1: 377-394.

\section{Vaginal fibrosarcoma in a cow}

B. Musal, P. Ulutas, A. Aydogan

Faculty of Veterinary Medicine, Adnan Menderes University, Isikli - Aydin, Turkey

Corresponding author: B. Musal. Tel: +90 2562470700

Email: bmusal@adu.edu.tr

Irish Veterinary Journal Volume 60 Number 7, 424-425, 2007

Vaginal fibrosarcomas are unusual mesenchymal tumours in cows. This report describes the clinical investigation, gross and histopathological findings, surgical treatment and postoperative course of a vaginal fibrosarcoma in a cow.

Fibropapillomas are the most commonly encountered type of tumours in the vagina and vulva of the cow. They are usually pedunculated and can be removed surgically. Although they do not cause infertility, they may be associated with dystocia (Noakes, 1996). Besides fibropapillomas, cases of squamous cell carcinoma, leiomyoma, fibroma, haemangioma, leiomyosarcoma and melanoma have also been reported in the vagina and vulva of cows (Yeruham et al., 1999). Fibrosarcomas can be found in any location of the body. However, they are unusual mesenchymal tumours of the bovine vagina (Kokuuslu et al., 1980; Deveci et al., 1988; Moulton, 1990). Fibromas, fibro-papillomas and fibrosarcomas have been reported as mushroom-shaped growths, and can be attached either by a broad base or by a long pedicle that allows part of the tumour to protrude from the vulva (Yeruham et al., 1999).

An eight-year-old Holstein Freisian cow was admitted for the evaluation of a pedunculated vaginal mass that was attached to the right lateral vaginal wall and partially protruded from the vagina (Figure 1). The mass had a wet surface with a mucoid, sanguinous discharge. Appetite was normal and the general physical examination revealed no other abnormalities.
Haematological examination prior to surgery revealed a leucocytosis. The total WBC count was elevated to 16.1 cells $/ \mu \mathrm{L}$ (range 4-12), with a high neutrophil count of 8090 cells/ $\mu \mathrm{L}$ (range 600-4000) and a lymphocyte count at the high end of physiological normal $(7490$ cells/ $\mu \mathrm{L}$; range 2500-7500). Other parameters, such as RBC, HGB and HCT were slightly below physiological range, which may be an indicator of mild anaemia due to the continuous haemorrhagic discharge from the mass. Serum biochemistry showed minor increases in ALT and creatinine but AST, total protein, triglycerides, phosphorus and AST were all within the normal range.

The cow was restrained and the tail bandaged. Local epidural anaesthesia was performed with the administration of $8 \mathrm{ml}$ of $2 \%$ lidocaine (Jetokain; Adeka, Turkey).

Additional local infiltration anaesthesia, within the vaginal mucosa surrounding the pedicle of the tumoral mass, was performed with the same anaesthetic agent using a volume of approximately $15-20 \mathrm{ml}$. Following anaesthesia, the vulva was retracted from either side with uterine forceps and the mass was revealed. An oval incision was made on the mucoasl surface at a distance of approximately $2 \mathrm{~cm}$ from the margin of the mass. The total length of incision was $15 \mathrm{~cm}$. Following this, blunt dissection with scissors was used to increase the depth of the incision without interfering with the edge of the mass. The major blood vessels were ligated where necessary. The defect created after the removal of the 